\title{
Imaging findings of multisystem inflammatory syndrome in children associated with COVID-19
}

\author{
Pablo Caro-Domínguez ${ }^{1}$ (1) María Navallas ${ }^{2} \cdot$ Lucia Riaza-Martin $^{3} \cdot$ Maryam Ghadimi Mahani $^{4}$. \\ Carlos F. Ugas Charcape ${ }^{5}$. Israel Valverde ${ }^{6}$. Felice $D^{\prime}$ Arco $^{7}$ • Seema Toso ${ }^{8}$ - Susan Cheng Shelmerdine $9,10,11$. \\ Joost van Schuppen ${ }^{12}$. Aurelio Secinaro ${ }^{13}$. Daniel Gräfe ${ }^{14} \cdot$ Marisol Camacho $^{15}$. Olaf Neth ${ }^{15} \cdot$ Hyun Woo Goo $^{16}$. \\ Christian J. Kellenberger ${ }^{17}$
}

Received: 31 July 2020 / Revised: 30 November 2020 / Accepted: 17 March 2021 / Published online: 27 April 2021

(C) The Author(s), under exclusive licence to Springer-Verlag GmbH Germany, part of Springer Nature 2021

\begin{abstract}
Background A hyperinflammatory immune-mediated shock syndrome has been recognised in children exposed to the severe acute respiratory syndrome coronavirus 2 (SARS-CoV-2), the virus that causes coronavirus disease 2019 (COVID-19).

Objective To describe typical imaging findings in children with multisystem inflammatory syndrome associated with COVID-19. Materials and methods During the first wave of the COVID-19 pandemic, imaging studies and clinical data from children treated for multisystem inflammatory syndrome were collected from multiple centres. Standardised case templates including demographic, biochemical and imaging information were completed by participating centres and reviewed by paediatric radiologists and paediatricians. Results We included 37 children (21 boys; median age 8.0 years). Polymerase chain reaction (PCR) testing was positive for SARS-CoV-2 in 15/37 (41\%) children and immunoglobulins in 13/19 children (68\%). Common clinical presentations were fever $(100 \%)$, abdominal pain $(68 \%)$, rash $(54 \%)$, conjunctivitis $(38 \%)$ and cough $(32 \%)$. Thirty-three children (89\%) showed laboratory or imaging findings of cardiac involvement. Thirty of the 37 children $(81 \%)$ required admission to the intensive care unit, with good recovery in all cases. Chest radiographs demonstrated cardiomegaly in $54 \%$ and signs of pulmonary venous hypertension/congestion in $73 \%$. The most common chest CT abnormalities were ground-glass and interstitial opacities $(83 \%)$, airspace consolidation (58\%), pleural effusion (58\%) and bronchial wall thickening (42\%). Echocardiography revealed impaired cardiac function in half of cases $(51 \%)$ and coronary artery abnormalities in 14\%. Cardiac MRI showed myocardial oedema in 58\%, pericardial effusion in $42 \%$ and decreased left ventricular function in $25 \%$. Twenty children required imaging for abdominal symptoms, the commonest abnormalities being free fluid (71\%) and terminal ileum wall thickening (57\%). Twelve children underwent brain imaging, showing abnormalities in two cases.

Conclusion Children with multisystem inflammatory syndrome showed pulmonary, cardiac, abdominal and brain imaging findings, reflecting the multisystem inflammatory disease. Awareness of the imaging features of this disease is important for early diagnosis and treatment.
\end{abstract}

Keywords Children $\cdot$ Coronavirus $\cdot$ COVID-19 $\cdot$ Imaging $\cdot$ Multisystem inflammatory syndrome $\cdot$ Radiology

\section{Introduction}

In early April, paediatricians from Europe and the United States detected an unexplained increase of cases with similarities to septic shock, myocarditis and Kawasaki-like syndrome in children [1-3]. They hypothesised a possible association

Pablo Caro-Domínguez pablocaro82@hotmail.com

Extended author information available on the last page of the article with current or recent infection by severe acute respiratory syndrome coronavirus 2 (SARS-CoV-2), the novel coronavirus that causes coronavirus disease 2019 (COVID-19). Since then, similar cases have been reported worldwide [4-7].

Two names are commonly used to describe this entity. The Royal College of Paediatric and Child Health (RCPCH) uses the term "paediatric inflammatory multisystem syndrome temporally associated with SARS-CoV-2" (PIMS-TS) [8], whereas the World Health Organization [9] and United States Centers for Disease Control and Prevention [10] refer to it as multisystem inflammatory syndrome in children (MIS- 
C). For this manuscript, we use the more widely used term, multisystem inflammatory syndrome in children. Diagnosis is complex and requires biochemical evidence of elevated inflammatory markers, absence of other microbial causes and evidence of organ dysfunction (e.g., hypotension, shock, myocardial dysfunction). The association with SARS-CoV-2 infection has been defined variably: while the RCPCH states that the child may or may not test positive for SARS-CoV-2, the WHO states that evidence of COVID-19 might include positive polymerase chain reaction (PCR), antigen test, serology or a history of contact with people with COVID-19 $[8,9]$. The temporal association with SARS-CoV-2 has been recognised internationally as the most important factor despite the variation in case definitions among organisations.

More than 1,000 cases had been reported worldwide at the time of this report [10], presenting with a broad spectrum of clinical findings including persistent fever, gastrointestinal symptoms and rash. Variable disease severity has been recognised, including shock and myocardial injury, requiring different levels of care [11,12]. Nevertheless, information on the imaging spectrum of this hyperinflammatory syndrome is still quite sparse and is mainly based on case reports and case series [13-15]. In this study, our objective was to describe typical imaging findings in children with multisystem inflammatory syndrome.

\section{Materials and methods}

Hospital Universitario Virgen del Rocío, Seville, Spain, granted ethical approval to study the imaging appearance of COVID-19 in children. We obtained parental/guardian approval for each case to share the anonymised data.

The European Society of Paediatric Radiology (ESPR) Cardiothoracic Taskforce conceived the study and members of multiple international radiologic societies were invited to submit anonymised demographic, clinical and imaging data from children with multisystem inflammatory syndrome at their institutions. We used a standardised questionnaire (Supplementary Material 1) to collect data, which were submitted to the office of the ESPR over a 4-week period, between 26 May 2020 and 21 June 2020. The most representative chest radiograph and the five most representative US, CT and MR images of each child were submitted by the physician collaborators on this study, specifying the day they were performed. The inclusion criteria were any patient younger than 18 years who fulfilled the case definition described by $\mathrm{RCPCH}$ for multisystem inflammatory syndrome in children (Table 1) [16].

Different groups of radiologists and subspecialised paediatricians reviewed the submitted medical data and imaging studies relating to their area of expertise. Clinical and laboratory data were reviewed by two paediatric rheumatologists and a paediatric cardiologist with 21,25 and 12 years of experience, respectively (M.C., O.N. and I.V.). Chest radiographs, CT, echocardiography and cardiac MRI were analysed by a group of five senior paediatric radiologists and a paediatric cardiologist with $2,8,8,11,20$ and 12 years of experience in paediatric chest imaging, respectively (M.N., J.V.S., A.S., M.G.M, C.J.K. and I.V.). Abdominal US, CT and MRI were evaluated by two paediatric radiologists, each with 10 years of experience in paediatric abdominal imaging (S.C.S. and S.T.). Cranial CT and MRI were analysed by a paediatric neuroradiologist and paediatric radiologist with 6 and 7 years of experience, respectively (F.D. and P.C.-D.). Not all imaging was available for review; in cases submitted without images, we used radiologic and echocardiography findings reported in the questionnaire.

The different groups of radiologists reviewed the images independently before comparing their results and reaching a consensus decision on the abnormalities, without disagreements. Descriptive analyses of the patient demographics, clinical and laboratory data, and imaging findings were performed and tabulated.

\section{Results}

\section{Clinical and laboratory findings}

In this case series, we included 37 children who met the criteria for multisystem inflammatory syndrome associated with COVID-19 (21 boys, 16 girls; median age 8.0 years, interquartile range [IQR] 3.3-10.3 years). Patient characteristics and clinical findings are summarized in Table 2 . We received cases from Spain $(n=12)$, France $(n=9)$, Iran $(n=8)$, Switzerland ( $n=4)$, Peru $(n=3)$ and Mexico $(n=1)$. SARS-

Table 1 The Royal College of Paediatrics and Child Health (RCPCH) case definition of paediatric multisystem inflammatory syndrome temporally associated with COVID-19 [15]

RCPCH definition of MIS-C

1. A child presenting with persistent fever, inflammation (neutrophilia, elevated CRP and lymphopenia) and evidence of single or multi-organ dysfunction (shock, cardiac, respiratory, renal, gastrointestinal or neurologic disorder) with additional features. This may include children fulfilling full or partial criteria for Kawasaki disease

2. Exclusion of any other microbial cause, including bacterial sepsis, staphylococcal or streptococcal shock syndromes, infections associated with myocarditis such as enterovirus

3. SARS-CoV-2 PCR testing may be positive or negative

COVID-19 coronavirus disease 2019, CRP C-reactive protein, $M I S-C$ multisystem inflammatory syndrome in children associated with COVID-19, $P C R$ polymerase chain reaction, $S A R S-C o V-2$ severe acute respiratory syndrome coronavirus 2 
CoV-2 PCR testing using nasopharyngeal swabs was positive for 14 children (38\%) and negative for 23, performed at median day 5 after symptom onset (IQR 3-6 days). Immunoglobulin $\mathrm{G}$ testing was performed on 20 children (54\%; median 7.5 days, IQR 5-13 days after symptom onset) and was positive for 13 of them (65\%), including 10 of 23 children with negative PCR. Thirteen children with negative PCR and immunoglobulin testing not performed had either family contact with a COVID-19 patient $(n=5)$ or lived in a region with high rate of infection ( $n=8$, Iran and France). All of them had the criteria described by the RCPCH [8]. PCR and serology testing are included in Table 3.

The most common clinical presentation was fever and gastrointestinal and respiratory symptoms, summarized in Table 2. Contact with SARS-CoV-2-positive family members was reported in 14 cases (38\%): 5 had positive immunoglobulin G (with negative PCR), 2 had positive PCR (serology not performed), 1 was positive for both tests, 2 were negative for both tests, 3 were negative for PCR (serology not obtained) and 1 was positive PCR obtained 3 weeks before the disease onset. Thirty of $37(81 \%)$ children required admission to the intensive care unit because of clinical deterioration, and one of them required extracorporeal membrane oxygenation (ECMO) for severe left ventricular dysfunction. All children recovered adequately after treatment, without fatalities. Only

Table 2 Summary of demographic and clinical findings in 37 children with multisystem inflammatory syndrome associated with coronavirus disease 2019 (COVID-19)

\begin{tabular}{ll}
\hline Demographics & Value \\
\hline Age & \\
All cases median & $8.0 \mathrm{y}$ \\
Interquartile range (range) & $3.3-10.3 \mathrm{y}$ \\
& $(3.0 \mathrm{~m}$ to $15.8 \mathrm{y})$ \\
Gender, $n(\%)$ & Boys, $21(57 \%)$ \\
& Girls, $16(43 / \%)$ \\
Family contact, $n(\%)$ & $14(38 \%)$ \\
Symptoms, $n(\%)$ & \\
Fever & $37(100 \%)$ \\
Abdominal pain & $25(68 \%)$ \\
Rash & $20(54 \%)$ \\
Conjunctivitis & $14(38 \%)$ \\
Cough & $12(32 \%)$ \\
Dyspnoea & $9(24 \%)$ \\
Vomiting & $5(14 \%)$ \\
Diarrhoea & $4(11 \%)$ \\
Headaches & $4(11 \%)$ \\
Lymphadenopathy & $4(11 \%)$ \\
Chest pain & $3(8 \%)$ \\
\hline
\end{tabular}

$m$ months, $y$ years
1/37 children ( $3 \%$ ) had a comorbidity, a 6-month-old boy with Down syndrome and atrioventricular septal defect.

Laboratory data measured in affected children showed a marked pro-inflammatory state reflected in high serum $\mathrm{C}$ reactive protein, procalcitonin, ferritin, interleukin-6, natriuretic peptide, troponin and D-dimer serum levels. In addition, and in support of the earlier, lymphopenia and hypoalbuminemia was noted. Blood tests for troponin were available in 32/ $37(86 \%)$ cases and were elevated in 28 patients. Natriuretic peptide was elevated in all 20/37 $(54.1 \%)$ patients tested. Laboratory data are summarized in Table 3.

\section{Cardiothoracic findings}

Cardiothoracic imaging results were reported in the questionnaire by the case submitters. Images with findings were available to the reviewers in $27 / 36$ cases $(75 \%)$ with chest radiographs, in $10 / 12$ cases $(83 \%)$ with chest CT, in $4 / 35$ cases (11\%) with echocardiography and in $10 / 12$ cases $(83 \%)$ with cardiac MRI. Thirty-three $(33 / 37,89 \%)$ children with multisystem inflammatory syndrome showed laboratory evidence or imaging findings of cardiac involvement.

Chest radiographs were obtained in all but one child (36/ $37,97 \%$ ) with the first image performed within the first few days after symptom onset (median 4 days, IQR 3-6 days,

Table 3 Pathological laboratory results of 37 children with multisystem inflammatory syndrome associated with coronavirus disease 2019 (COVID-19)

\begin{tabular}{ll}
\hline Laboratory values (normal values) & Median (IQR) \\
\hline Haemoglobin $(11.1-15.5 \mathrm{~g} / \mathrm{dL})$ & $10.6(9.2-11.2)$ \\
Neutrophils $\left(1.5-7.0 \times 10^{9} / \mathrm{L}\right)$ & $7.6(6.0-10.7)$ \\
Lymphocytes $\left(1.2-3.5 \times 10^{9} / \mathrm{L}\right)$ & $0.9(0.5-1.7)$ \\
CRP $(<5 \mathrm{mg} / \mathrm{L})$ & $139(92.3-150.8)$ \\
Procalcitonin $(<0.25 \mathrm{mcg} / \mathrm{L})$ & $9.8(4.1-34.4)$ \\
Ferritin $(14-125 \mathrm{mcg} / \mathrm{L})$ & $603(458-1,144)$ \\
IL-6 $(<7 \mathrm{pg} / \mathrm{mL})$ & $336(216.5-428.0)$ \\
NT-proBNP $(<112 \mathrm{ng} / \mathrm{L})$ & $7,206(4,403-13,631)$ \\
Troponin $(0-14 \mathrm{ng} / \mathrm{L})$ & $41(22.3-112.5)$ \\
D-dimer $(<0.5 \mathrm{mg} / \mathrm{L})$ & $4.2(2.8-9.9)$ \\
Albumin $(38-54 \mathrm{~g} / \mathrm{l})$ & $29(26-32.3)$ \\
SARS-CoV-2 testing & \\
PCR positive, $n=14$ & Ig G positive, $n=3$ \\
& Ig G negative, $n=1$ \\
& Ig G n/a, $n=10$ \\
PCR negative, $n=23$ & Ig G positive, $n=10$ \\
& Ig G negative, $n=6$ \\
& Ig G n/a, $n=7$ \\
\hline
\end{tabular}

$C R P$ C-reactive protein, $I g G$ immunoglobulin G, $I L-6$ interleukin 6, IQR interquartile range $\left(\min \mathrm{Q}_{1}-\max \mathrm{Q}_{3}\right)$, n/a not available, NT-proBNP Nterminal pro b-type natriuretic peptide, $P C R$ polymerase chain reaction 


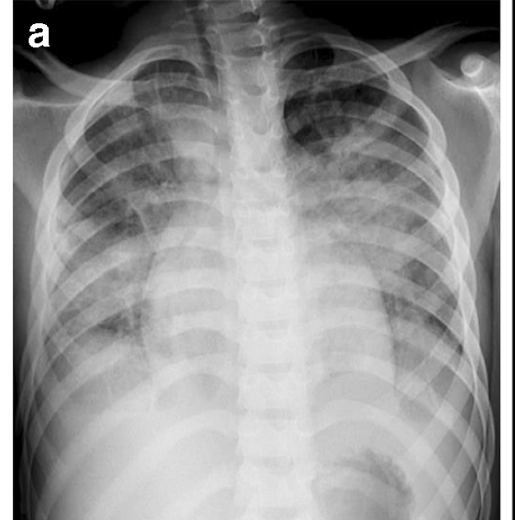

Fig. 1 Cardiothoracic and abdominal imaging findings in a 10-year-old boy presenting with fever, cough, rash and abdominal pain, with positive anti-SARS-CoV-2 (severe acute respiratory syndrome coronavirus 2) immunoglobulin G. a Anteroposterior chest radiograph shows bilateral

range 1-15 days). Indications for performing a chest radiograph were mostly fever $(13 / 36,36 \%)$, to check the position of an endotracheal tube $(9 / 36,25 \%)$, intensive care unit admission $(8 / 36,22 \%)$, dyspnoea $(4 / 36,11 \%)$, hypoxemia $(1 / 36$, $3 \%$ ) and cardiac shock $(1 / 36,3 \%)$. Anteroposterior chest images showed cardiomegaly in 20/36 (56\%) and signs of variable pulmonary venous hypertension/congestion in $27 / 36$ (75\%) children (Figs. 1 and 2). Signs of pulmonary inflammation included increased central bronchovascular markings in $22 / 36(61 \%)$, peripheral interstitial reticular pattern in $7 / 36$ (19\%), perihilar ground-glass opacities in 9/36 (25\%) (Figs. 2 and 3) and airspace consolidation in 10/36 children (28\%) (Fig. 1). Pleural effusion was found in 6/36 (17\%) (Fig. 1). Chest radiographs were considered normal without any relevant findings in 6/37 (16\%) children.

Chest CT was performed in 12/37 (32\%) children between day 2 and day 30 (median 6 days, IQR 5-9 days), in 7 without intravenous contrast agent and in 5 with contrast agent. Only

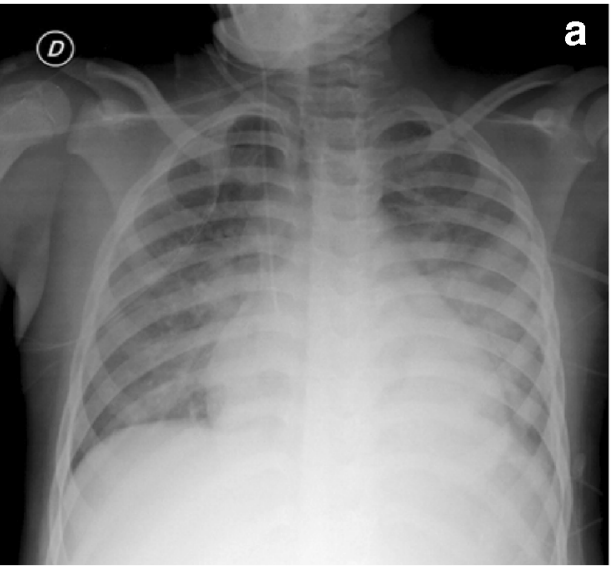

Fig. 2 Cardiothoracic findings in a 6-year-old boy presenting with impaired biventricular function on echocardiography 6 days after the initial onset of fever, abdominal pain, rash and conjunctivitis. AntiSARS-CoV-2 (severe acute respiratory syndrome coronavirus 2 ) total immunoglobulins $\mathrm{G}, \mathrm{A}$ and $\mathrm{M}$ were positive. a Anteroposterior chest one of these contrast-enhanced CTs had a technique sufficient for assessing the coronary arteries; this confirmed the mild coronary artery dilatation previously detected on echocardiography. Indications for performing chest CT were poor response to treatment $(6 / 12,50 \%)$, refractory hypoxemia $(4 / 12,33 \%)$, dyspnoea $(1 / 12,8 \%)$ and multiorgan dysfunction $(1 / 12,8 \%)$. The most common findings were ground-glass opacity in 10/12 (83\%) (Fig. 3) and interstitial reticular or reticulonodular densities in 9/12 $(75 \%)$ cases. Bronchial wall thickening was seen in 5/12 (42\%) cases. Bilateral airspace consolidation was present in 7/12 cases (58\%) while nodularappearing lesions were seen in one case $(1 / 12,8 \%)$ (Fig. 3). Small pleural effusions were evident in 7/12 (58\%) and pericardial effusion in $4 / 12$ cases $(23 \%)$. Ten children had pleural effusion either on chest radiography or CT (27\%). No pulmonary embolism was reported.

Echocardiography was also obtained relatively early after symptom onset (median 5 days, IQR 4-8 days, range 1-

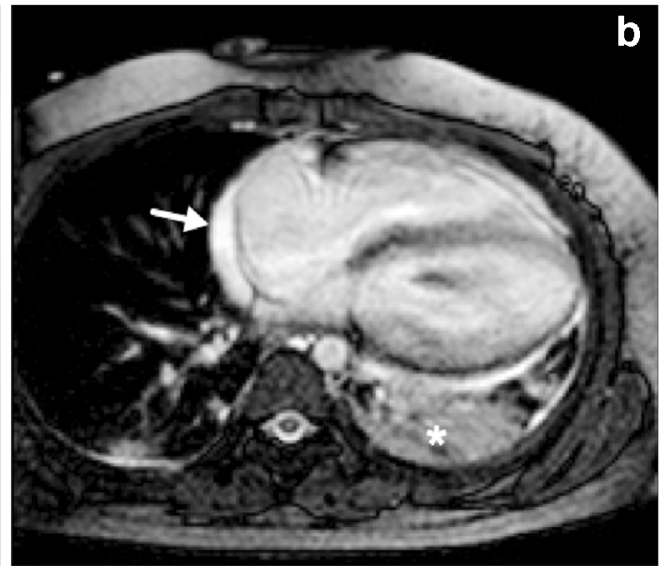

radiograph shows cardiomegaly, dilated azygos vein, small left pleural effusion, bilateral airspace consolidation and ground-glass opacities as signs of pulmonary oedema. b Axial balanced turbo field echo cardiac MR image shows consolidation in the left lower lobe (asterisk), small pericardial effusion (arrow) and bilateral small pleural effusion 
Fig. 3 Cardiothoracic findings in a 12-year-old girl with fever, abdominal pain, rash, diarrhoea and hypotension who required admission to the intensive care unit. a Initial anteroposterior (AP) radiograph on day 3 reveals focal left upper lobe consolidation and ground-glass opacity. b On day 5 she developed refractory hypoxemia and her AP chest radiograph reveals bilateral diffuse ground-glass opacities, bilateral pleural effusion, focal consolidation in the right upper lobe and interval enlargement of the cardiac silhouette, suggestive of acute respiratory distress syndrome. $\mathbf{c}, \mathbf{d}$ Axial (c) and coronal (d) images from a chest $\mathrm{CT}$ performed the same day as (b) shows bilateral upper lobe ground-glass opacity, septal thickening, right pleural effusion, and nodular consolidation/ pulmonary nodules in both lower lobes (arrows)

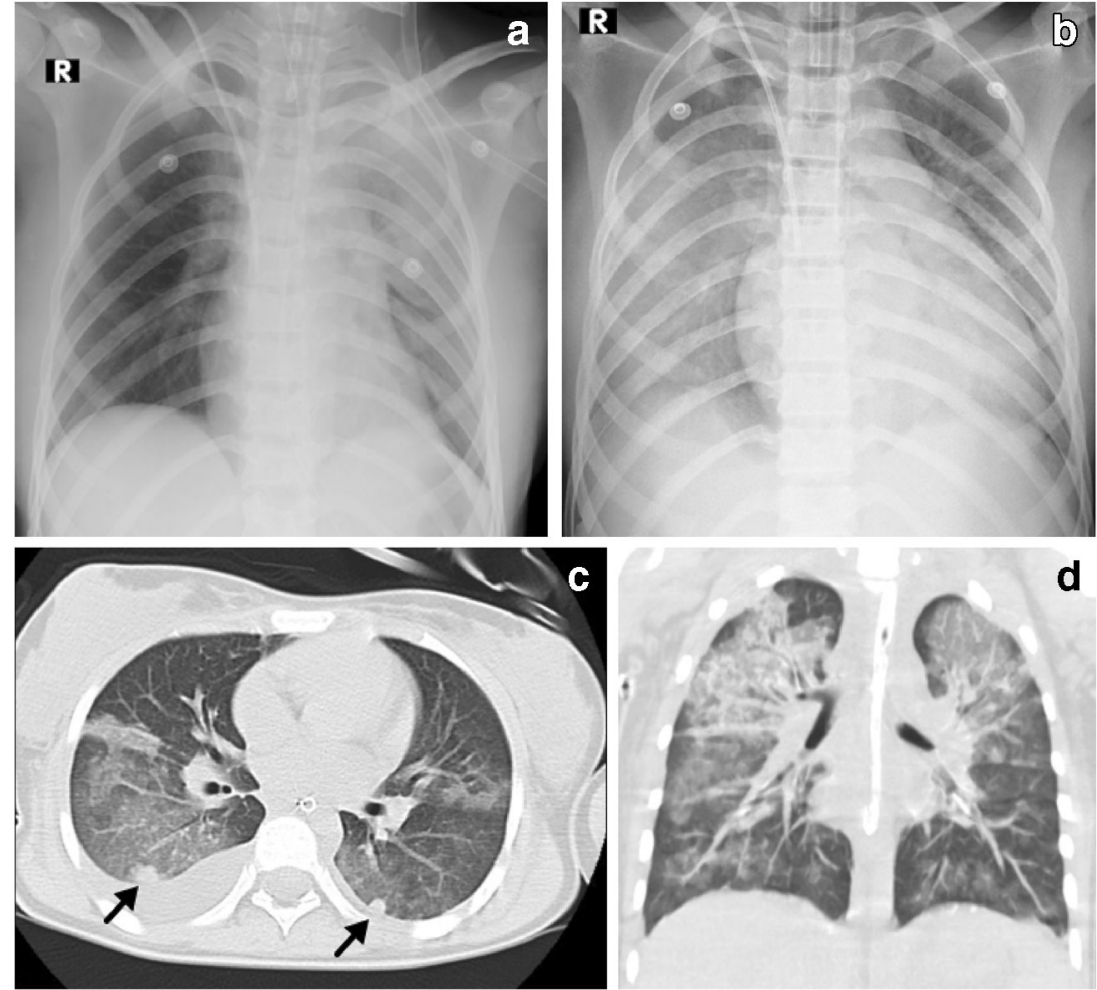

30 days) in 35/37 (95\%) children. Echocardiography was performed to evaluate ventricular function and to rule out myocarditis when there was laboratory evidence for cardiac involvement or abnormal findings on electrocardiogram. Nineteen $(19 / 35,54 \%)$ children showed signs of impaired cardiac function, with decreased left ventricular ejection fraction of $50 \%$ or less in 16/35 (46\%) cases, atrioventricular valve regurgitation in $4 / 35(11 \%)$ cases and signs of increased central venous pressure in $3 / 35(9 \%)$ cases. In all cases with left ventricular dysfunction, left ventricular function had improved at the time of hospital discharge. Pericardial effusion was reported in 6/35 cases (17\%). Coronary artery abnormalities were detected in 5/35 cases (14\%), including dilatation of the proximal segments in 3 cases (Fig. 4) and increased wall echogenicity in 2 cases. No coronary artery aneurysms were reported.

Cardiac MRI was performed in 12/37 cases (32\%) between 5 days and 100 days after symptom onset (median 25 days, IQR 9-24 days), to study the cause of the ventricular dysfunction seen on echocardiography or to rule out myocarditis. In

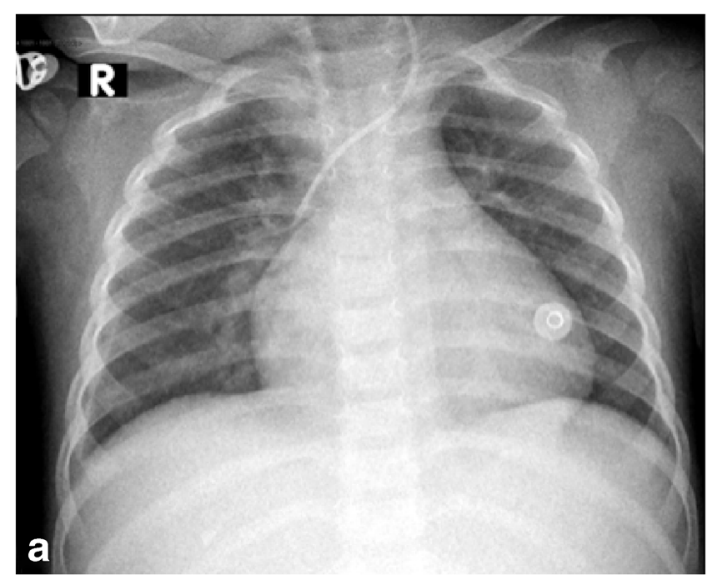

Fig. 4 Coronary artery dilatation in a 2-year-old girl presenting with fever, abdominal pain and rash. SARS-CoV-2 (severe acute respiratory syndrome coronavirus 2) polymerase chain reaction (PCR) and immunoglobulins $\mathrm{G}$ and $\mathrm{M}$ were positive. a Anteroposterior chest radiograph shows cardiomegaly, increased bronchovascular markings

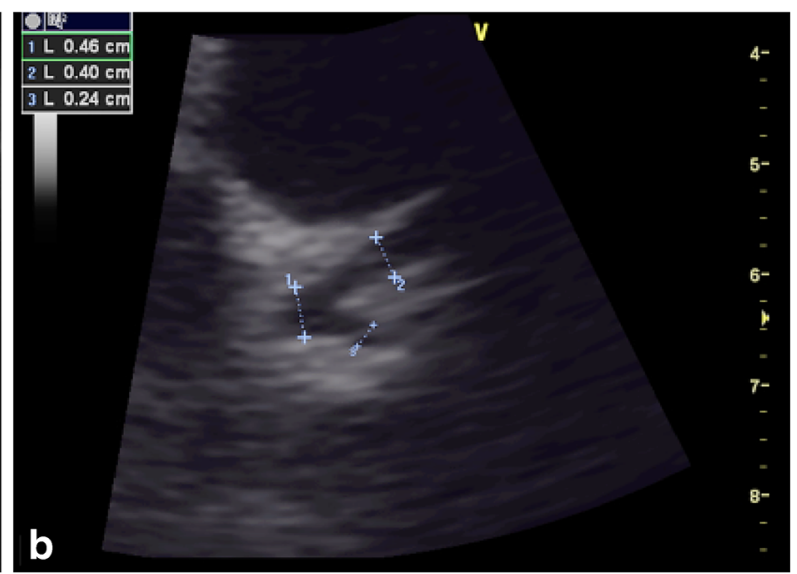

and small right pleural effusion. b Modified parasternal short-axis view echocardiograph shows dilatation of the left main coronary and the left anterior descending arteries measuring $4 \mathrm{~mm}$ (z score of +2.4$)$ and $4 \mathrm{~mm}$ (z score of +2.6 ), respectively (cursors) 

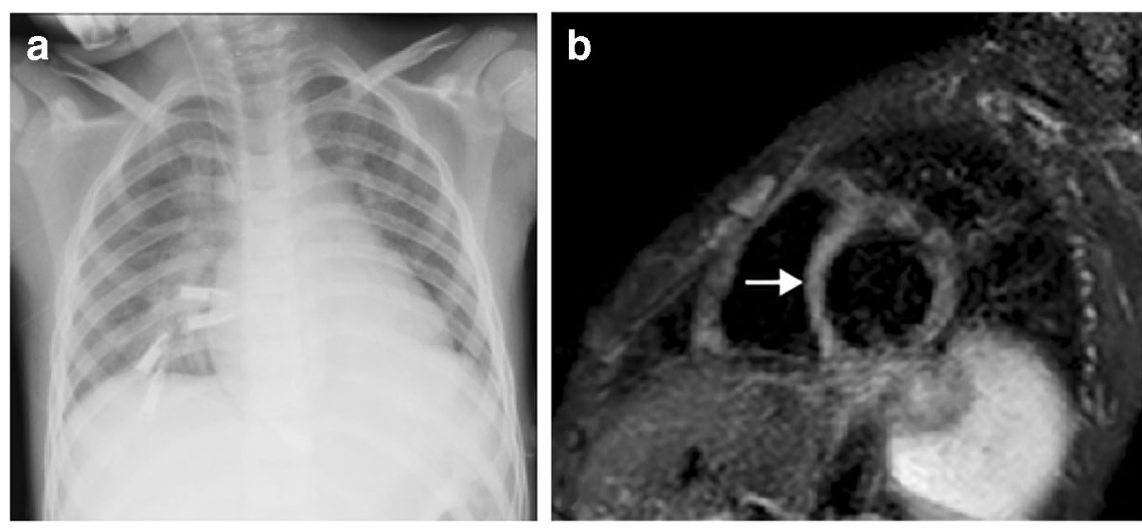

Fig. 5 Cardiomegaly in a 9-year-old boy initially presenting with fever, cough, abdominal pain and rash. SARS-CoV-2 (severe acute respiratory syndrome coronavirus 2) polymerase chain reaction (PCR) was positive. a Anteroposterior radiograph at day 7 shows cardiomegaly and perihilar opacities consistent with pulmonary oedema. b, c Cardiac MRI at day 24 to investigate cardiomegaly and repolarisation abnormalities shows

six cases with decreased left ventricular ejection fraction $(50 \%$ or less) on echocardiography, the ventricular function had normalised at the time of MRI. Three of the 12 MRI studies (25\%) showed decreased left ventricular ejection fraction, with severe left ventricular dilatation in 1 case. Seven of the $12(58 \%)$ children showed varying degrees of myocardial oedema (Figs. 5 and 6), with additional focal early and late myocardial gadolinium enhancement in one case (Fig. 6). Small pericardial effusion was seen in 5/12 (42\%) children (Figs. 2 and 6). No myocardial perfusion defects were detected in 6/6 cases in which first-pass perfusion was performed. No coronary artery abnormalities were reported. Cardiothoracic findings are summarized in Table 4.

\section{Abdominal findings}

Twenty (20/37, 54\%) children ( 7 girls, 13 boys, mean age 7.3 years) with multisystem inflammatory syndrome received at least one type of abdominal imaging study during their assessment. Seventeen of the $20(85 \%)$ underwent abdominal US, 6 (30\%) underwent abdominal CT and $2(10 \%)$ underwent abdominal MRI. Abdominal US images were available to the reviewers in 6/6 (100\%) cases, with abdominal CT in $2 / 2(100 \%)$ and abdominal MRI in $1 / 2(50 \%)$. Indications for performing the imaging were mostly abdominal pain $(14 / 20,70 \%)$, distension $(2 / 20,10 \%)$ or related to sepsis $(4 / 20,20 \%)$. Abdominal US was performed on average at 7.8 days after onset of symptoms (range 2-30 days), whilst CT and MRI were performed after 4.8 days (range 2-8 days). Abdominal findings are summarized in Table 5.

Six $(6 / 20,30 \%)$ children did not have any abdominal abnormalities on imaging. Of those with findings $(14 / 20,70 \%)$, the commonest abnormality was free fluid (10/14, 71\%), followed by bowel wall thickening $(8 / 14,57 \%)$, which normalised ventricular volumes with normal function, residual mild myocardial oedema with myocardial to skeletal muscle signal ratio of 2:1 on short-axis short tau inversion recovery (STIR) image (arrow in b), but without corresponding late gadolinium enhancement on short-axis inversion recovery fast gradient echo image (c)

commonly affected the terminal ileum (7/8, 88\%) (Fig. 7). The whole colon was involved in only one case $(1 / 8,13 \%)$ (Fig. 1). Other less common findings included hepatomegaly (5/14, 36\%), right iliac fossa lymphadenopathy $(4 / 14,29 \%)$, gallbladder wall oedema $(3 / 14,21 \%)$ (Fig. 7$)$, gallbladder sludge $(2 / 14,14 \%)$, periportal oedema $(2 / 14,14 \%)$ and splenomegaly $(2 / 14,14 \%)$. One child was found to have haemorrhagic cystitis $(1 / 14,7 \%)$. There were no features of appendicitis or bowel or solid visceral infarction. Two children had clinical and laboratory findings of acute renal failure without significant abnormalities on abdominal US.

\section{Brain findings}

Twelve children with multisystem inflammatory syndrome needed brain imaging (9 MRI and $3 \mathrm{CT}$ ) during their hospitalization (32\%); 3/12 cases showed abnormal findings $(25 \%)$. Indications for performing brain imaging were headache, seizure, persistent drowsiness, decreased level of consciousness, papilledema, confusion, signs of encephalopathy, or post cardio-respiratory arrest. Head CT images were available for the reviewers in $1 / 3(33 \%)$ cases and head MRI in 4/9 (44\%) cases. A 15-year-old boy with septic shock, renal failure and myocarditis showed leptomeningeal enhancement of the right precentral sulcus on day 9 (Fig. 8). A 5-year-old girl with fever, abdominal pain, dehydration and confusion showed foci of restricted diffusion in the splenium of the corpus callosum (Fig. 9). Finally, an 11-month-old girl with congenital heart disease demonstrated generalised atrophy of the cerebrum with decreased volume and abnormal signal of the white matter, most likely related to her longstanding cardiac disease rather than to COVID-19, without acute findings noted. None of the children needed spinal cord imaging. 

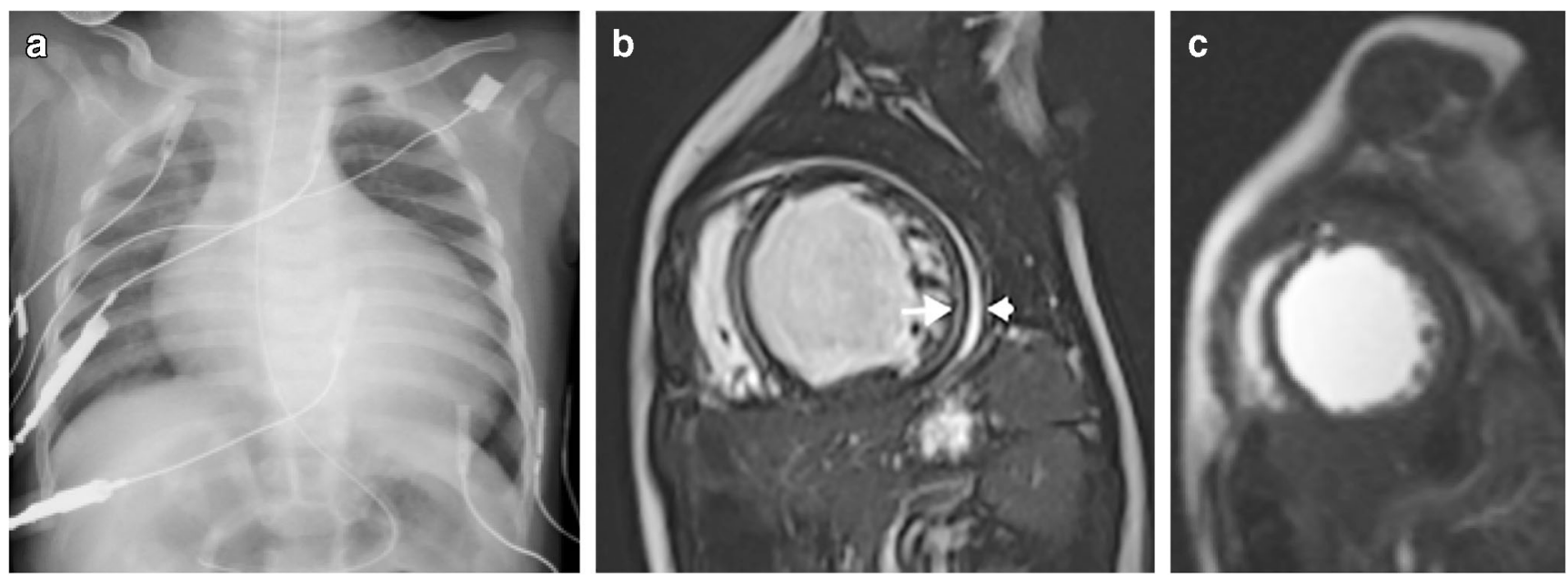

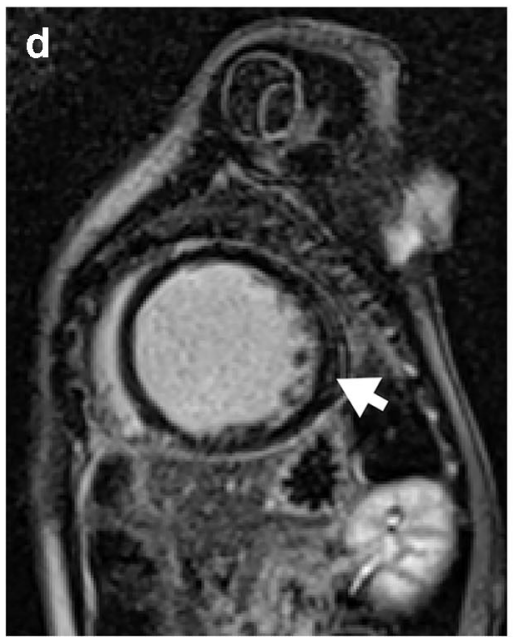

Fig. 6 Cardiac failure in a 6-month-old boy with positive SARS-CoV-2 (severe acute respiratory syndrome coronavirus 2) polymerase chain reaction (PCR) test. a Anteroposterior chest radiograph shows cardiomegaly. b-f MRI. Short-axis cardiac MR images show dilated left ventricle with increased signal intensity of the myocardium on

\section{Discussion}

This article shows that multisystem inflammatory syndrome in children has distinct imaging features that can be recognised. Children diagnosed with this disease showed imaging findings of cardiac, pulmonary, abdominal and to a lesser extent neurologic inflammation, most of which were temporary and associated with good clinical recovery, highlighting the multisystem nature of this immunemediated disease.

Signs of pulmonary congestion, enlarged cardiomediastinal silhouette, and pleural effusion in children with previous SARS-CoV-2 infection might suggest multisystem inflammatory syndrome. The described chest radiography findings include bronchial wall thickening, ground-glass opacity, consolidation, pulmonary oedema, interstitial opacities and pleural fluid, which can be the result of pulmonary inflammation and vasculitis $[5,8,17]$. However, most of these findings could also be related to impaired cardiac function or treatment of a critically ill child (e.g., in resuscitation).

While the percentage and type of the chest radiography findings in our study are similar to the reported abnormalities in the literature, in many articles the exact type of abnormality in chest radiograph was either not described or not separated from the $\mathrm{CT}$ analysis, or the chest radiograph interpretations were not performed by a radiologist [5, 11, 18-25]. Hameed et al. [13] had a detailed description of the radiologic findings in their study and they reported bronchial wall thickening in $34 \%$ (12/35) of their cases. In our study, this was the most common abnormality on chest radiograph, seen in $54 \%$ of our cases. Eleven of Hameed et al.'s [13] 12 cases progressed rapidly to perihilar airspace opacification, associated with either cardiac dysfunction or fluid resuscitation. Focal airspace consolidation was seen in $14 \%$ of our cohort, similar to Hameed's study. Presence of pleural effusion in our group was $27 \%$ of cases, higher than Hameed's cohort of $11 \%$. 
Table 4 Cardiac and pulmonary imaging findings in 37 children with multisystem inflammatory syndrome associated with coronavirus disease 2019 (COVID-19)

\begin{tabular}{|c|c|c|c|c|}
\hline Cardiac/pulmonary findings & Radiography & Echocardiography & $\mathrm{CT}$ & Cardiac MRI \\
\hline Study frequency & $36 / 37(97 \%)$ & $35 / 37(95 \%)$ & $12 / 37(32 \%)$ & $12 / 37(32 \%)$ \\
\hline \multicolumn{5}{|l|}{ Cardiac findings } \\
\hline Cardiomegaly & $20 / 36(56 \%)$ & & $1 / 12(8 \%)$ & $1 / 12(8 \%)$ \\
\hline Pulmonary congestion/oedema & $27 / 36(75 \%)$ & & $8 / 12(67 \%)$ & \\
\hline Impaired ventricular function & & $16 / 35(46 \%)$ & & $3 / 12(25 \%)$ \\
\hline Atrioventricular valve insufficiency & & $4 / 35(11 \%)$ & & \\
\hline Increased central venous pressure & & $3 / 35(9 \%)$ & & \\
\hline Pericardial effusion & & $6 / 35(17 \%)$ & $4 / 12(33 \%)$ & $5 / 12(42 \%)$ \\
\hline Coronary artery abnormality & & $5 / 35(14 \%)$ & $1 / 12(8 \%)$ & \\
\hline Myocardial oedema & & & & $7 / 12(58 \%)$ \\
\hline \multicolumn{5}{|l|}{ Lung findings } \\
\hline Increased, ill-defined central broncho-vascular markings ${ }^{\mathrm{a}}$ & $22 / 36(61 \%)$ & & $5 / 12(42 \%)$ & \\
\hline Peripheral reticular pattern/septal thickening & $7 / 36(19 \%)$ & & $9 / 12(75 \%)$ & \\
\hline Ground-glass opacity & $9 / 36(25 \%)$ & & $10 / 12(83 \%)$ & \\
\hline Airspace opacity/consolidation & $10 / 36(28 \%)$ & & $7 / 12(58 \%)$ & \\
\hline Pleural effusion & $6 / 36(17 \%)$ & & $7 / 12(58 \%)$ & \\
\hline Nodular consolidation on $\mathrm{CT}$ & & & $1 / 12(8 \%)$ & \\
\hline
\end{tabular}

${ }^{\mathrm{a}}$ Includes bronchial wall thickening, peribronchial cuffing

All chest $\mathrm{CT}$ studies in our cohort were abnormal. Most CT findings could be interpreted either as inflammatory or secondary to cardiac function impairment. The distribution of our CT chest findings is different from that of Hameed et al. [13]. In our study, the most common findings were ground-glass $(83 \%)$ and interstitial (75\%) opacities (Figs. 2 and 3), whilst basal consolidation and collapse were the most frequent abnormalities in the Hameed cohort, present in $33 \%$ of their cases [13]. In our cohort, airspace consolidation was seen in $73 \%$ of cases. Hameed et al. [13] reported one child with multiple small, rounded consolidations with surrounding ground-glass halos. We also had a case with multiple rounded consolidations and solid nodules, which did not have a halo (Fig. 3). Pulmonary nodules with or without ground-glass halo have been reported in inflammatory lung disease including Kawasaki disease [17].

It is important to note that respiratory tract symptoms are not considered a leading feature of multisystem inflammatory syndrome. A child with positive PCR and respiratory symptoms more likely has COVID-19 and not multisystem inflammatory syndrome. Nonetheless, in our series of 37 children fulfilling the criteria for multisystem inflammatory syndrome, $32 \%$ had cough and $24 \%$ had dyspnoea. Furthermore, respiratory symptoms have also been described in this disease [26].

The findings of this series suggest that cardiac involvement in children with multisystem inflammatory syndrome appears to include a myocarditis-type disease besides the Kawasakitype coronary artery disease [12, 27]. Blondiaux et al. [14] found in their cohort of four children that myocardial oedema and hyperaemia were the commonest abnormalities, similar to our study. These findings might reflect indirect myocardial injury from acute severe inflammation rather than direct cardiomyocyte damage such as in viral myocarditis or as reported

Table 5 Abdominal imaging findings in 20 children with multisystem inflammatory syndrome associated with coronavirus disease 2019 (COVID-19) and abdominal symptoms

\begin{tabular}{ll}
\hline Abdominal findings & $n(\%)$ \\
\hline Study frequency & \\
$\quad$ Ultrasound & $17 / 20(85 \%)$ \\
CT & $6 / 20(30 \%)$ \\
MRI & $2 / 20(10 \%)$ \\
No findings & $6 / 20(30 \%)$ \\
Free fluid & $10 / 14(71 \%)$ \\
Bowel wall thickening & $8 / 14(57 \%)$ \\
$\quad$ Terminal ileum & $7 / 8(88 \%)$ \\
$\quad$ Colon & $1 / 8(13 \%)$ \\
Hepatomegaly & $5 / 14(36 \%)$ \\
Right iliac fossa lymphadenopathy & $4 / 14(29 \%)$ \\
Gallbladder wall oedema & $3 / 14(21 \%)$ \\
Gallbladder sludge & $2 / 14(14 \%)$ \\
Periportal oedema & $2 / 14(14 \%)$ \\
Splenomegaly & $2 / 14(14 \%)$ \\
\hline
\end{tabular}




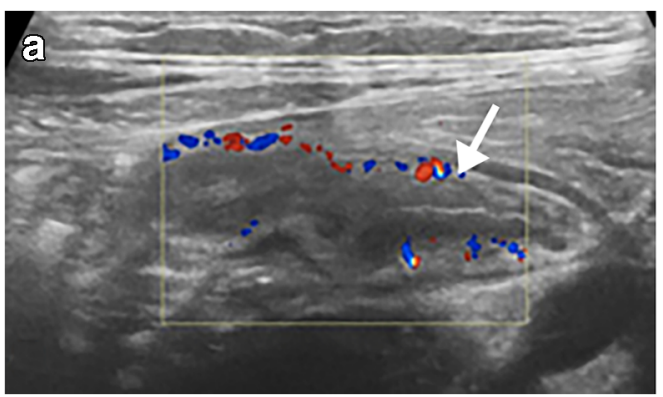

\section{b}

Fig. 7 Abdominal findings in a 13-year-old girl presenting with fever, dyspnoea and abdominal pain. Her anti-SARS-CoV-2 (severe acute respiratory syndrome coronavirus 2) immunoglobulin $\mathrm{G}$ was positive. a-c Abdominal US was performed 4 days after symptom onset.
Transverse colour Doppler US image of the right iliac fossa region demonstrates ileal thickening (arrow in a); sagittal view of the right upper abdomen shows gallbladder wall oedema (arrow in b), and sagittal view of the pelvis demonstrates free fluid (star in $\mathbf{c}$ ) in adults with COVID-19 [28, 29]. In our series, only one child showed evidence of myocardial injury on late gadolinium enhancement despite most of the cases being performed several days after symptom onset.

Echocardiography, which was obtained early after symptoms onset, showed signs of impaired cardiac function in half the children; this had resolved in most of them by the time the cardiac MRI was performed at a median interval of 25 days after clinical presentation. This finding is supported by work by Blondiaux et al. [14], who found transient systolic dysfunction in all patients, and by Ramcharan et al. [25], who showed recovery of ventricular function in most of their cohort by the time of discharge. Coronary artery abnormalities were detected by echocardiography in $14 \%$, including proximal dilatation and increased wall echogenicity. Toubiana et al. [5] showed higher incidence of coronary artery abnormalities in their cohort of 21 children and adolescents presenting with features of Kawasaki disease, with $38 \%$ showing coronary artery abnormalities. Similar to Toubiana et al. [5], Belhadjer et al. [30] and Ramcharan et al.'s [25] series, no coronary aneurysms were identified in our cohort. The increased echogenicity of the coronary arteries has also been previously described in the literature $[4,25]$.
The pathophysiology for cardiac involvement remains to be clarified but could be caused by a post-viral immunological reaction rather than direct viral organ damage $[24,31]$. This theory is supported by ventricular function improvement and significant decrease of inflammatory biomarkers following intravenous immunoglobulin therapy [32]. In our series only one child had pre-existing congenital heart disease. Other comorbidities such as asthma and overweight have been described by Belhadjer et al. [30] as risk factor for acute heart failure in children with multisystem inflammatory syndrome.

The presence of abdominal findings in the setting of multisystem inflammatory syndrome has recently been reported in a few case series. Hameed et al. [13] found that in their cohort of 19 children who underwent abdominal US imaging, free fluid was the commonest abnormality in more than half the cases, similar to our study. Approximately $20 \%$ of their cases had bowel wall thickening, also predominantly involving the terminal ileum, apart from one case of localised caecal wall thickening [13]. This finding is supported by work by Tullie et al. [33], who found that in all eight children with multisystem inflammatory syndrome and gastrointestinal symptoms, the terminal ileum wall was thickened. In our series,
Fig. 8 Brain MRI findings in a 15year-old boy with meningism 9 days after the initial onset of fever, rash, conjunctivitis and abdominal pain. His anti-SARS-CoV-2 (severe acute respiratory syndrome coronavirus 2) immunoglobulins $\mathrm{G}$ and $\mathrm{M}$ were positive. a, b Axial (a) and sagittal (b) post-contrast 3-D fluid-attenuated inversion recovery MR images show leptomeningeal enhancement (arrows) in the right pre-central sulcus
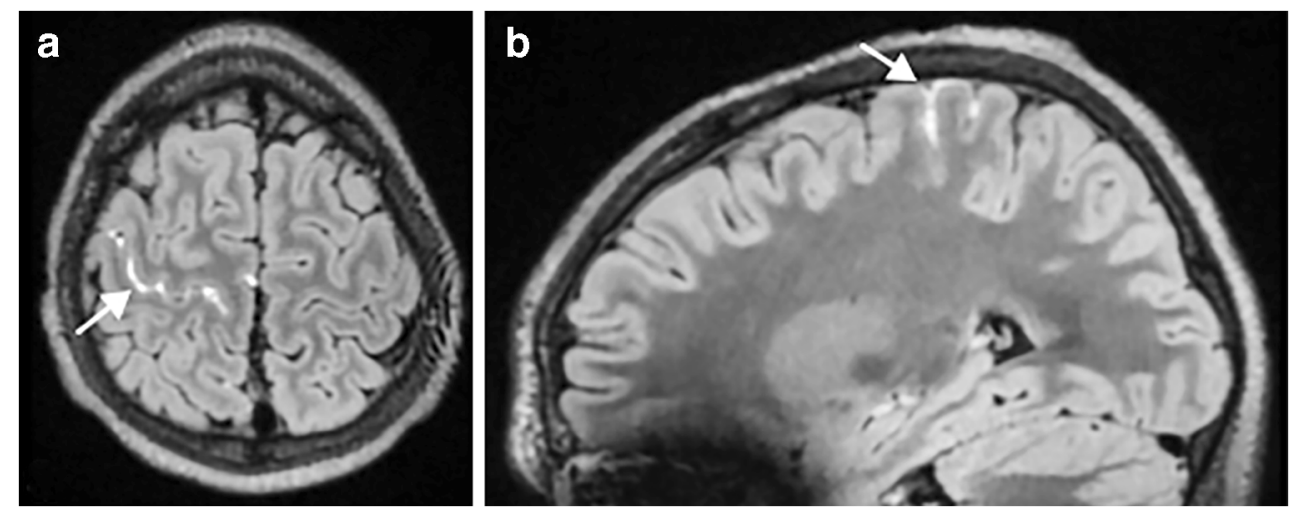
Fig. 9 Brain MRI findings in a 5year-old girl with altered level of consciousness 5 days after the initial onset of fever, abdominal pain and dehydration. Her antiSARS-CoV-2 (severe acute respiratory syndrome coronavirus 2) immunoglobulin $G$ was positive. a, b Axial diffusionweighted MR image $(b=1,000 ; \mathbf{a})$ and corresponding apparent diffusion coefficient (ADC) map (b) show restricted diffusion in the splenium of the corpus callosum (arrow)
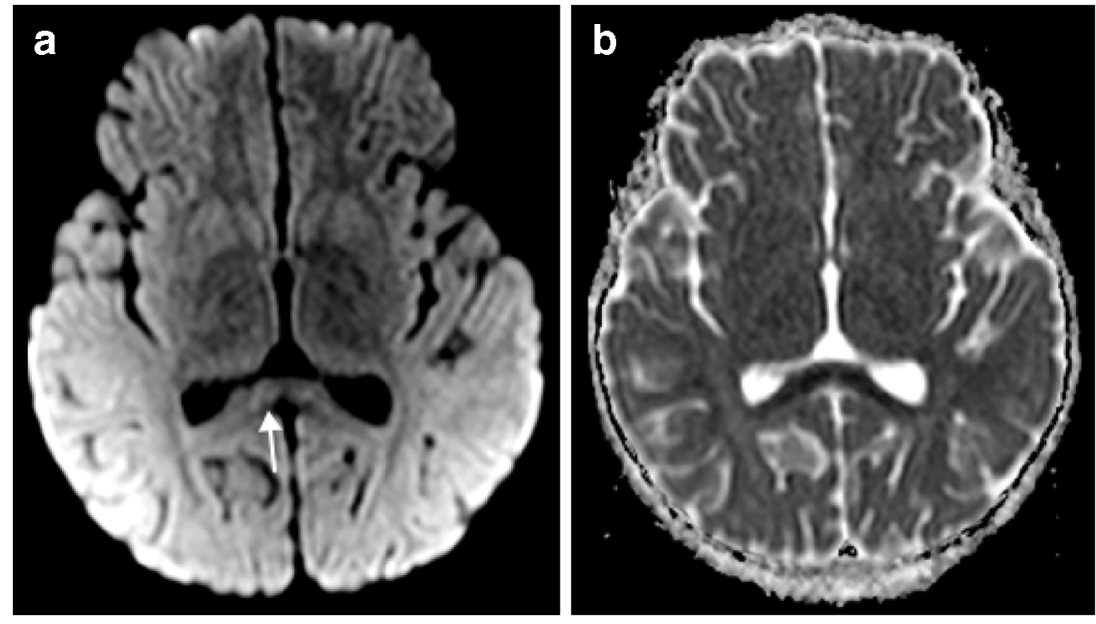

terminal ileal involvement was seen in $7 / 8(88 \%)$ children, reflecting similar localisation of inflammatory change.

The pathophysiology for bowel involvement remains uncertain but could be caused by a delayed immune-mediated response to the virus because most patients in previous series had positive serology and negative PCR [13]. The abundance of lymphatic tissue and Peyer patches in the distal ileum might be one reason this region is particularly susceptible to inflammatory change [34]. Clinicians and radiologists should therefore remain vigilant of this important association in this clinical setting to avoid overcalling inflammatory bowel disease or assuming that abdominal pain is from appendicitis. Alternatively, when terminal ileal inflammation is detected in a child with persistent fevers and abnormal laboratory findings, then multisystem inflammatory syndrome could be raised as a possible differential diagnosis.

In contrast to adult bowel findings in COVID-19 [35] and the findings in the paediatric series by Hameed et al. [13], we did not identify any children with bowel or solid visceral infarction. Nevertheless, it is worth mentioning that splenic infarction in children is a recognised complication of inflammatory vasculitis and Kawasaki disease. Previous reports have highlighted the "COVID-19-induced coagulopathy" effect [36], and therefore signs of ischemia or infarction should be sought out when imaging any body part in a child with suspected SARS-CoV-2 exposition.

Two children with multisystem inflammatory syndrome in our series showed acute neuroimaging findings: restricted diffusion of the splenium of the corpus callosum in a 5-year-old girl (Fig. 9) and leptomeningeal enhancement in a 15-year-old boy (Fig. 8). Foci of abnormal signal in the splenium of the corpus callosum have been reported to be characteristic (although not specific) of children with COVID-19 [37] and are probably related to cytokine activation [38]. Leptomeningeal enhancement appears to be a common feature in adults with COVID-19 with neurologic symptoms [39], reflecting an immune reaction of the meninges against SARS-CoV-2 infection.

This study has several limitations. It is a retrospective study with cases collected from multiple institutions and countries. Collection of cases with representative imaging poses a selection bias; nonetheless, our results demonstrate typical imaging findings in children with multisystem inflammatory syndrome associated with COVID-19 but not their frequency in all children affected. The heterogeneous image quality and protocols in the numerous centres, the probably variable expertise of the physicians who performed the imaging studies, data collection by questionnaire without detailed definitions of imaging findings, and our review of only selected images have an unknown impact on detection and frequency of individual findings reported in our series. Some laboratory data were not available. Because follow-up radiographs were not available for review, we cannot comment on the evolution of findings. We decided to use the case definition from the RCPCH as inclusion criteria for multisystem inflammatory syndrome in children because it was available at the time this study was conceived. Eight children included in our study did not have positive testing or a family contact and would have been excluded using the WHO case definition. However, they came from areas of high prevalence of disease and the attending physicians diagnosed and treated them as cases of multisystem inflammatory syndrome.

\section{Conclusion}

In this multicentre retrospective study, children diagnosed with multisystem inflammatory syndrome associated with COVID-19 showed imaging findings of cardiac, pulmonary, abdominal and to a lesser extent neurologic inflammation, most of which were temporary and associated with good clinical recovery. The clinical presentation 
was supported by abnormal laboratory or imaging findings, comparable with a pro-inflammatory state of the host. Chest imaging findings of pulmonary congestion, enlarged cardio-mediastinal silhouette, and pleural effusion found in children with serology evidence of previous SARS-CoV-2 infection should suggest the diagnosis of multisystem inflammatory syndrome. Echocardiography and cardiac MRI help to characterise the cardiac disease, mainly myocarditis and less often coronary abnormalities in our series. Ascites and ileal wall thickening might be seen on abdominal imaging. Brain involvement might appear as restricted diffusion of the corpus callosum or focal leptomeningeal enhancement. There is need for a greater awareness of the multisystem appearances of this immune-mediated disease, with a low threshold for further (preferably non-ionising) imaging where required.

Supplementary Information The online version contains supplementary material available at https://doi.org/10.1007/s00247-021-05065-0.

Acknowledgements We thank Anca Cociuban from the European Society of Paediatric Radiology Office for her help in collecting the cases for this article. We also acknowledge all of the collaborators of the European Society of Paediatric Radiology Cardiothoracic Task Force: Judith Almanza, Paediatric Radiology, Hospital Infantil de México Federico Gómez, Mexico City, Mexico; Carlos Alvarez Murillo, Pediatric Cardiology, Instituto Nacional de Salud del Niño San Borja, Lima, Perú; Soheila Alyasin, Allergy Research Center, Shiraz University of Medical Sciences, Shiraz, Iran; Aline Carsin-Vu, Paediatric Radiology, American Memorial Hospital, CHU de Reims, Reims, France; David Coca Robinot, Paediatric Radiology, Hospital Universitario 12 de Octubre, Madrid, Spain; Marirosa Cristallo Lacalamita, Paediatric Radiology, EOC, IIMSI Istituto di Imaging della Svizzera Italiana, Switzerland; Shabnam Hajiani Ghotbabadi, Paediatric Rheumatology Diseases, Shiraz University of Medical Sciences, Shiraz, Iran; Pooya Iranpour, Medical Imaging Research Center, Shiraz University of Medical Sciences, Shiraz, Iran; Reza Jalli, Medical Imaging Research Center, Shiraz University of Medical Sciences, Shiraz, Iran; Karmella Kamali, Medical Imaging Research Center, Shiraz University of Medical Sciences, Shiraz, Iran; María Martínez León, Radiología Pediátrica, Hospital Materno-Infantil, Hospital Regional Universitario, Málaga, Spain; Arnaud Maurin, Paediatric Radiology, Hôpitaux Universitaires de Marseille Timone, France; Franklin Mendoza Torres, Pediatric Intensive Care Unit, Instituto Nacional de Salud del Niño San Borja, Lima, Perú; Marta Pelaz Esteban, Paediatric Radiology, Hospital Universitario Marqués de Valdecilla, Santander, Spain; Blandine Petain, Paediatric Radiology, Hôpitaux Universitaires de Marseille Timone, France; Gholamreza Pouladfar, Professor Alborzi Clinical Microbiology Research Center, Shiraz University of Medical Sciences, Shiraz, Iran; Miguel Rasero, Paediatric Radiology, Hospital Universitario 12 de Octubre, Madrid, Spain; Constance Riviere, Paediatric Radiology, Hôpitaux Universitaires de Marseille Timone, France; Amie Saeed, Department of Paediatrics, Division of Paediatric Intensive Care Unit, Shiraz University of Medical Sciences, Shiraz, Iran; Anahita Sanaei Dashti, Professor Alborzi Clinical Microbiology Research Center, Shiraz University of Medical Sciences, Shiraz, Iran; Eslam Shorafa, Department of Paediatrics, Paediatric Intensive Care Unit, Shiraz University of Medical Sciences, Shiraz, Iran; Slivadji Vingadassalom,
Paediatric Radiology, Hôpitaux Universitaires de Marseille Timone, France.

\section{Declarations}

Conflicts of interest None

\section{References}

1. Levin M (2020) Childhood multisystem inflammatory syndrome - a new challenge in the pandemic. N Engl J Med 383:393-395

2. Jones VG, Mills M, Suarez D et al (2020) COVID-19 and Kawasaki disease: novel virus and novel case. Hosp Pediatr 10: 537-540

3. Pediatric Critical Care Society (2020) NHS warning. Retweet. https://twitter.com/PICSociety/status/1254508725227982848

4. Riphagen S, Gomez X, Gonzalez-Martinez C et al (2020) Hyperinflammatory shock in children during COVID-19 pandemic. Lancet 395:1607-1608

5. Toubiana J, Poirault C, Corsia A et al (2020) Kawasaki-like multisystem inflammatory syndrome in children during the COVID-19 pandemic in Paris, France: prospective observational study. BMJ 369:m2094

6. Verdoni L, Mazza A, Gervasoni A et al (2020) An outbreak of severe Kawasaki-like disease at the Italian epicentre of the SARS-CoV-2 epidemic: an observational cohort study. Lancet 395:1771-1778

7. Whittaker E, Bamford A, Kenny J et al (2020) Clinical characteristics of 58 children with a pediatric inflammatory multisystem syndrome temporally associated with SARS-CoV-2. JAMA 324: 259-269

8. Royal College of Paediatric and Child Health (2020) Paediatric multisystem inflammatory syndrome temporally associated with COVID-19 (PJIMS) - guidance for clinicians. RCPCH website. https://www.rcpch.ac.uk/resources/guidance-paediatricmultisystem-inflammatory-syndrome-temporally-associatedcovid-19. Accessed 12 Feb 2021

9. World Health Organization (2020) Multisystem inflammatory syndrome in children and adolescents with COVID-19. Scientific brief. WHO website. https://www.who.int/publications/i/item/ multisystem-inflammatory-syndrome-in-children-and-adolescentswith-covid-19. Accessed 12 Feb 2021

10. Centers for Disease Control and Prevention (2020) Multisystem inflammatory syndrome in children (MIS-C) associated with coronavirus disease 2019 (COVID-19). CDC website. https:// emergency.cdc.gov/han/2020/han00432.asp. Accessed 12 Feb 2021

11. Dufort EM, Koumans EH, Chow et al (2020) Multisystem inflammatory syndrome in children in New York State. N Engl J Med 383:347-358

12. Feldstein LR, Rose EB, Horwitz et al (2020) Multisystem inflammatory syndrome in U.S. children and adolescents. N Engl J Med 383:334-346

13. Hameed S, Elbaaly H, Reid CEL et al (2020) Spectrum of imaging findings on chest radiographs, US, CT, and MRI images in multisystem inflammatory syndrome in children (MIS-C) associated with COVID-19. Radiology 298:E1-E10

14. Blondiaux E, Parisot P, Redheuil A et al (2020) Cardiac MRI of children with multisystem inflammatory syndrome associated with COVID-19. Radiology 297:E283-E288

15. Blumfield E, Levin TL, Kurian J et al (2021) Imaging findings in multisystem inflammatory syndrome in children (MIS-C) associat- 
ed with coronavirus disease (COVID-19). AJR Am J Roentgenol 216:507-517

16. Freeman AF, Crawford SE, Finn LS et al (2003) Inflammatory pulmonary nodules in Kawasaki disease. Pediatr Pulmonol 36: 103-106

17. Umezawa T, Saji T, Matsuo N, Odagiri K (1989) Chest X-ray findings in the acute phase of Kawasaki disease. Pediatr Radiol 20:48-51

18. Cheng C, Li C, Zhao T et al (2020) COVID-19 with rheumatic diseases: a report of 5 cases. Clin Rheumatol. https://doi.org/10. 1007/s10067-020-05160-x

19. Cheung EW, Zachariah P, Gorelik M et al (2020) Multisystem inflammatory syndrome related to COVID-19 in previously healthy children and adolescents in New York City. JAMA 324:294-926

20. Ng KF, Kothari Y, Bandi S et al (2020) COVID-19 multisystem inflammatory syndrome in three teenagers with confirmed SARSCoV-2 infection. J Med Virol 92:2880-2886

21. Kaushik S, Aydin SI, Derespina KR et al (2020) Multisystem inflammatory syndrome in children (MIS-C) associated with SARSCoV-2 infection: a multi-institutional study from New York City. J Pediatr 224:24-29

22. Rodriguez-Gonzalez M, Rodriguez-Campoy P, Sanchez-Codez M et al (2020) New onset severe right ventricular failure associated with COVID-19 in a young infant without previous heart disease. Cardiol Young 30:1346-1349

23. Pain CE, Felsenstein S, Cleary G et al (2020) Novel paediatric presentation of COVID-19 with ARDS and cytokine storm syndrome without respiratory symptoms. Lancet Rheumatol 2:E376E379

24. Pouletty M, Borocco ON et al (2020) Paediatric multisystem inflammatory syndrome temporally associated with SARS-CoV-2 mimicking Kawasaki disease (Kawa-COVID-19): a multicentre cohort. Ann Rheum Dis 79:999-1006

25. Ramcharan T, Nolan O, Lai CY et al (2020) Paediatric inflammatory multisystem syndrome: temporally associated with SARSCoV-2 (PIMS-TS): cardiac features, management and short-term outcomes at a UK tertiary paediatric hospital. Pediatr Cardiol 41: 1391-1401

26. Ahmed M, Advani S, Moreira A et al (2020) Multisystem inflammatory syndrome in children: a systematic review. EClinicalMedicine 26:100527

27. Theocharis P, Wong J, Pushparajah K et al (2020) Multimodality cardiac evaluation in children and young adults with multisystem inflammation associated with COVID-19. Eur Heart J Cardiovasc Imaging. https://doi.org/10.1093/ehjci/jeaa212

28. Kim IC, Kim JY, Ha K, Han S (2020) COVID-19-related myocarditis in a 21-year-old female patient. Eur Heart J 41:1859

29. Inciardi RM, Lupi L, Zaccone G et al (2020) Cardiac involvement in a patient with coronavirus disease 2019 (COVID-19). JAMA Cardiol 5:819-824

30. Belhadjer Z, Méot M, Bajolle F et al (2020) Acute heart failure in multisystem inflammatory syndrome in children (MIS-C) in the context of global SARS-CoV-2 pandemic. Circulation 142:429436

31. Zhu H, Rhee J-W, Cheng P et al (2020) Cardiovascular complications in patients with COVID-19: consequences of viral toxicities and host immune response. Curr Cardiol Rep 22:32

32. Grimaud M, Starck J, Levy M et al (2020) Acute myocarditis and multisystem inflammatory emerging disease following SARSCoV-2 infection in critically ill children. Ann Intensive Care 10:69

33. Tullie L, Ford K, Bisharat M et al (2020) Gastrointestinal features in children with COVID-19: an observation of varied presentation in eight children. Lancet Child Adores Health 4:e19-e20

34. Sivit CJ, Newman KD, Chandra RS (1993) Visualization of enlarged mesenteric lymph nodes at US examination. Pediatr Radiol 23:471-475

35. Bhayana R, Som A, Li MD et al (2020) Abdominal imaging findings in COVID-19: preliminary observations. Radiology 297: E207-E215

36. Barnes GD, Burnett A, Allen A et al (2020) Thromboembolism and anticoagulant therapy during the COVID-19 pandemic: interim clinical guidance from the anticoagulation forum. J Thromb Thrombolysis 50:72-81

37. Abdel-Mannan O, Eyre M, Löbel U et al (2020) Neurologic and radiographic findings associated with COVID-19 infection in children. JAMA Neurol 77:1-6

38. Starkey J, Kobayashi N, Numaguchi Y, Moritani T (2017) Cytotoxic lesions of the corpus callosum that show restricted diffusion: mechanisms, causes, and manifestations. Radiographics 37: $562-576$

39. Purcell Y, Lecler A, Saragoussi E et al (2020) Neurologic involvement of patients with coronavirus disease 2019: making the most of MRI. Radiology 297:E239

Publisher's note Springer Nature remains neutral with regard to jurisdictional claims in published maps and institutional affiliations. 


\section{Affiliations}

\section{Pablo Caro-Domínguez ${ }^{1}$ (D) María Navallas $^{2}$ - Lucia Riaza-Martin ${ }^{3} \cdot$ Maryam Ghadimi Mahani $^{4}$.}

Carlos F. Ugas Charcape ${ }^{5}$ - Israel Valverde ${ }^{6}$. Felice D'Arco ${ }^{7}$. Seema Toso ${ }^{8}$. Susan Cheng Shelmerdine $9,10,11$. Joost van Schuppen ${ }^{12}$. Aurelio Secinaro ${ }^{13}$. Daniel Gräfe ${ }^{14} \cdot$ Marisol Camacho $^{15}$. Olaf Neth ${ }^{15} \cdot$ Hyun Woo Goo ${ }^{16}$. Christian J. Kellenberger ${ }^{17}$

1 Department of Radiology, Section of Pediatric Radiology, Hospital Universitario Virgen del Rocío, Avenida Manuel Siurot s/n., CP 41013 Seville, Spain

2 Department of Radiology, Section of Pediatric Radiology, Hospital Universitario 12 de Octubre, Madrid, Spain

3 Department of Radiology, Section of Pediatric Radiology, Hospital Universitario Vall d'Hebron, Barcelona, Spain

4 Department of Radiology, Section of Pediatric Radiology, University of Michigan, Ann Arbor, MI, USA

5 Department of Imaging, Instituto Nacional de Salud del Niño San Borja, Lima, Peru

6 Pediatric Cardiology and Cardiovascular Pathology Unit, Virgen del Rocio University Hospital, Institute of Biomedicine of Seville (IBIS), University of Seville, Seville, Spain

7 Department of Radiology, Neuroradiology Unit, Great Ormond Street Hospital for Children, NHS Foundation Trust, London, UK

Department of Diagnostic Imaging, Section of Pediatric Radiology, Geneva Children's Hospitals, Geneva, Switzerland
9 Department of Clinical Radiology, Great Ormond Street Hospital NHS Foundation Trust, London, UK

10 UCL Great Ormond Street Institute of Child Health, London, UK

11 NIHR Great Ormond Street Hospital Biomedical Research Centre, London, UK

12 Department of Radiology and Nuclear Medicine, Amsterdam UMC, University of Amsterdam, Amsterdam, The Netherlands

13 Department of Imaging, Bambino Gesù Children's Hospital, IRCCS, Rome, Italy

14 Department of Pediatric Radiology, University Hospital of Leipzig, Leipzig, Germany

15 Paediatric Infectious Diseases, Rheumatology and Immunology Unit, Hospital Universitario Virgen del Rocío, Institute of Biomedicine of Seville, Seville, Spain

16 Department of Radiology and Research Institute of Radiology, Asan Medical Center, University of Ulsan College of Medicine, Seoul, South Korea

17 Diagnostic Imaging, University Children's Hospital Zurich, Zurich, Switzerland 